\title{
Dapagliflozin Ameliorates the Formation and Progression of Experimental Abdominal Aortic Aneurysms by Reducing Aortic Inflammation in Mice
}

\author{
Haole Liu, ${ }^{1,2}$ Panpan Wei, ${ }^{2}$ Weilai Fu, ${ }^{3}$ Congcong Xia, ${ }^{2}$ Yankui Li $\mathbb{D},{ }^{3}$ Kangli Tian, ${ }^{1}$ \\ Yafeng Li, ${ }^{2}$ Daxin Cheng, ${ }^{2}$ Jiaying Sun, ${ }^{1}$ Yangwei $\mathrm{Xu},{ }^{1}$ Ming Lu, ${ }^{1}$ Boyu Xu, ${ }^{2}$ Yali Zhang $\mathbb{D}^{2}$ \\ Rong Wang, ${ }^{2}$ Weirong Wang, ${ }^{2}$ Baohui Xu $\mathbb{D}^{4},{ }^{4}$ Enqi Liu $\mathbb{D}^{2},{ }^{2}$ and Sihai Zhao $\mathbb{D}^{1,2}$ \\ ${ }^{1}$ Institute of Cardiovascular Science, Translational Medicine Institute, Xi'an Jiaotong University Health Science Center, Xi'an, \\ Shaanxi 710061, China \\ ${ }^{2}$ Laboratory Animal Center, Xi'an Jiaotong University, Xi'an, Shaanxi 710061, China \\ ${ }^{3}$ Department of Vascular Surgery, The Second Hospital of Tianjin Medical University, Tianjin 300211, China \\ ${ }^{4}$ Division of Vascular Surgery, Department of Surgery, Stanford University School of Medicine, Stanford, CA 94305, USA
}

Correspondence should be addressed to Sihai Zhao; sihaizhao@xjtu.edu.cn

Received 11 December 2021; Revised 3 January 2022; Accepted 10 January 2022; Published 27 January 2022

Academic Editor: Qi Yu

Copyright (c) 2022 Haole Liu et al. This is an open access article distributed under the Creative Commons Attribution License, which permits unrestricted use, distribution, and reproduction in any medium, provided the original work is properly cited.

\begin{abstract}
Background. Dapagliflozin, a sodium glucose transporter protein-2 (SGLT-2) inhibitor, reduces the risk for cardiovascular diseases. However, the influence of dapagliflozin on nondissecting abdominal aortic aneurysms (AAAs) remains unclear. Methods. AAAs were created in male C57BL/6 mice via intra-aortic porcine pancreatic elastase (PPE) infusion. Mice were daily treated with dapagliflozin ( 1 or $5 \mathrm{mg} / \mathrm{kg}$ body weight) or an equal volume of vehicle through oral gavage beginning one day prior to PPE infusion for 14 days. To investigate its translational value, dapagliflozin or vehicle was also administered to mice with existing AAAs in another cohort. Aortic diameters were measured prior to (day 0 for baseline) and 14 days after PPE infusion. After sacrifice, mice aortae were collected, and following histological analyses were performed. Results. Dapagliflozin treatment significantly reduced aneurysmal aortic expansion following PPE infusion as compared to vehicle treatment especially at $5 \mathrm{mg} /$ $\mathrm{kg}$ body weight (approximately $21 \%$ and $33 \%$ decreases in 1 and $5 \mathrm{mg} / \mathrm{kg}$ treatment groups, respectively). The dose-dependent attenuation of AAAs by dapagliflozin was also confirmed on histological analyses. Dapagliflozin remarkably reduced aortic accumulation of macrophages, $\mathrm{CD}^{+}{ }^{\mathrm{T}}$ cells, and B cells particularly following dapagliflozin treatment at $5 \mathrm{mg} / \mathrm{kg}$. Dapagliflozin treatment also markedly attenuated medial SMC loss. Though the difference was not significant, dapagliflozin treatment tended to attenuate $\mathrm{CD}^{+} \mathrm{T}$ cells and elastin degradation. Dapagliflozin treatment at $5 \mathrm{mg} / \mathrm{kg}$ caused a $53 \%$ reduction in neovessel density. Furthermore, dapagliflozin treatment mitigated further progress of existing AAAs. Conclusion. Dapagliflozin treatment ameliorated PPE-induced AAAs by inhibiting aortic leukocytes infiltration and angiogenesis.
\end{abstract}

\section{Introduction}

Abdominal aortic aneurysm (AAA) is a life-threaten vascular disease with approximately $80 \%$ mortality when ruptured $[1,2]$. Although mechanisms by which AAAs form and progress remain incompletely understood, inflammation has been considered as a central player in AAA pathogenesis $[3,4]$. Leukocytes, including T cells, macrophages, B cells, and mast cells play crucial roles in AAA disease [5-7].
Developing the strategies for lowering the incidence, or slowing the progression, of AAAs, is critical for effective disease management. Thus, pharmacological therapies for AAAs have attracted more attention in AAA research.

Diabetes is negatively associated with the formation and progression of clinical and experimental AAAs, with potential involvement of diabetes per se or diabetic medications [8-11]. Metformin, a widely used antidiabetic drug, suppressed experimental AAAs and reduced enlargement 

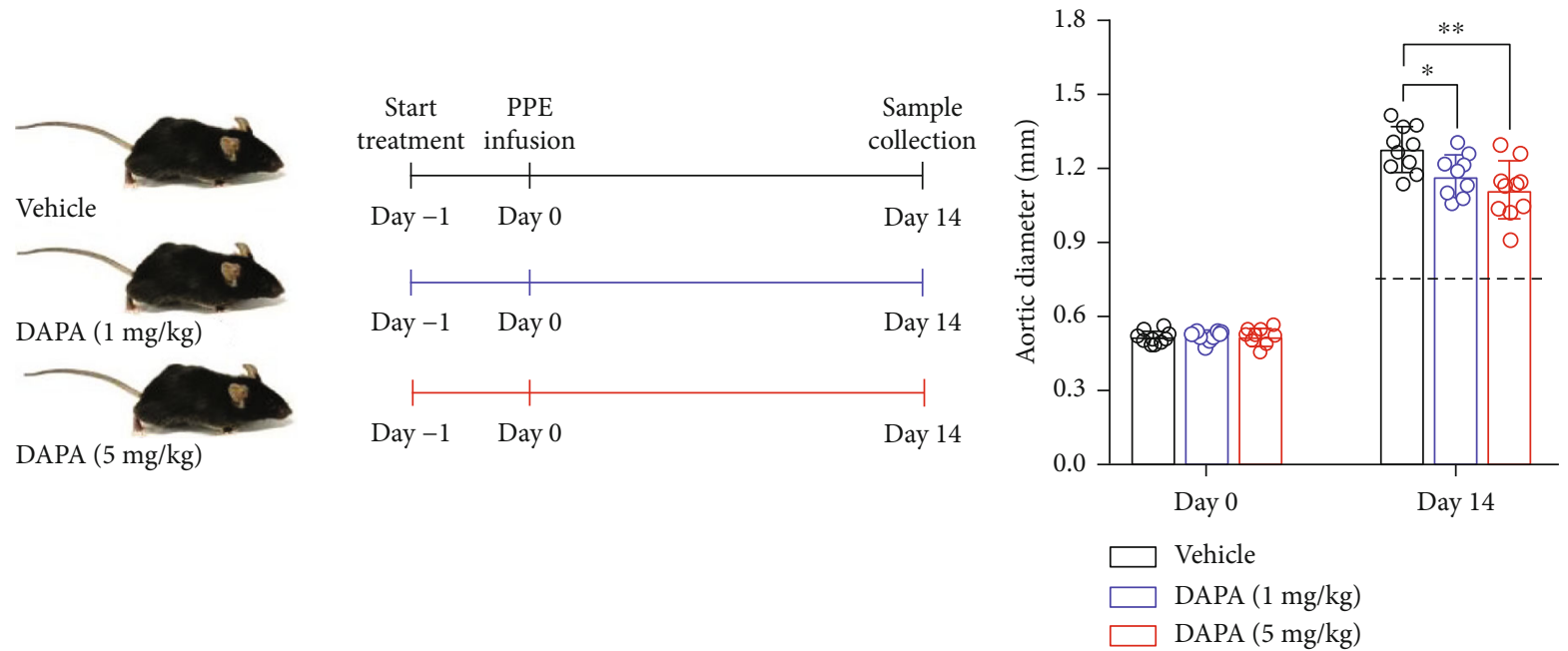

(a)

(b)

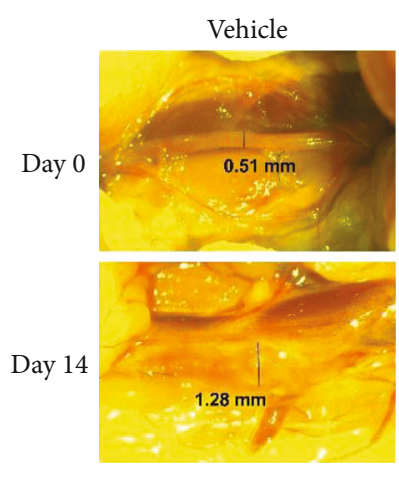

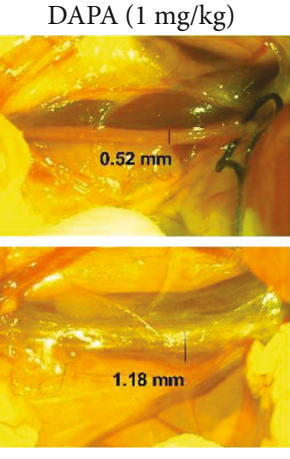

(c)

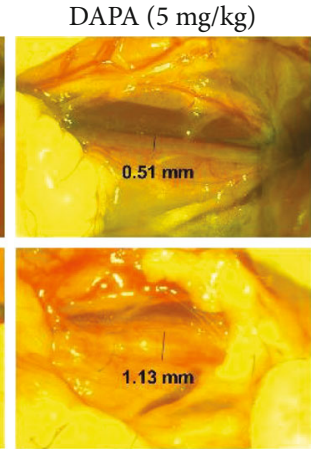

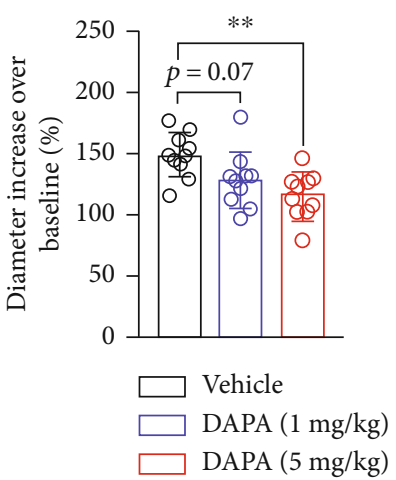

(d)

Figure 1: Dapagliflozin treatment suppresses experimental AAAs. (a) Study design: male C57BL/6 mice were given dapagliflozin (1 or 5 mg/ $\mathrm{kg}$ body weigh) or vehicle by gavage for 14 days ( $n=10$ mice/group). (b) Mean and SD of aortic diameters at the baseline level (day 0$)$ and 14 days after PPE infusion. Dotted line: the average aortic diameter following PBS infusion $(0.8 \mathrm{~mm})$. Two-way ANOVA followed by two group comparison, ${ }^{*} p<0.05$ and ${ }^{* *} p<0.01$ between two groups. (c) Representative abdominal aortic images at the baseline (day 0 ) and 14 days after PPE infusion. (d) Change in aortic diameter (percent increase over the baseline level). One-way ANOVA followed by two group comparison, ${ }^{* *} p<0.01$ between two groups. DAPA: dapagliflozin.

rate of clinical AAAs [11-15]. Other oral antidiabetic drugs, including sulfonylureas, thiazolidinediones, and dipeptidylpeptidase 4 inhibitors, have also been reported to lower AAA incidence or attenuate AAA enlargement [16]. These previous studies suggest that some antidiabetes drugs may hold the potential to limit AAA progression.

Inhibitors to sodium-glucose transporter protein-2 (SGLT-2) for treating type 2 diabetes have been shown to reduce the risk for cardiovascular morbidities [17, 18]. SGLT-2 inhibitor reportedly suppressed aortic dissection and dilation [19]. However, questions remain whether SGLT-2 inhibition influences experimental AAAs. In a previous study, SGLT-2 inhibitor attenuated dissecting AAAs in hyperlipidemic mice following angiotensin II (Ang II) infusion [19]. Whether SGLT-2 inhibitors influence nondissecting AAAs in alternative AAA models such as elastase infusion model has not been investigated. The previous studies focused on the preventive effect of SGLT-2 inhibitors on AAAs by administering the inhibitor prior to AAA induction. Thus, it is not known whether the SGLT-2 inhib- itor contains the progression of existing AAAs. In clinical trials, elective surgical AAA repair in patients with a diameter of $4.0-5.4 \mathrm{~cm}$ is not beneficial [20]. There is unmet clinical demand for innovating nonsurgical pharmacological therapies for limiting the continuous expansion and thus rupture of small AAAs [20, 21].

In this study, a SGLT-2 inhibitor dapagliflozin was administered to normoglycemic mice to evaluate the impact on AAA formation and progression induced by elastase infusion. Our study indicates that dapagliflozin treatment ameliorates the formation and progression of experimental AAAs partly by reducing aortic inflammation, neoangiogenesis, and preserving aortic structural integrity.

\section{Materials and Methods}

2.1. Mice. C57BL/6 mice were bred, maintained, and housed in the Laboratory Animal Center of Xi'an Jiaotong University, Xi'an, China. All experimental protocols were approved by the Laboratory Animal Administration Committee of 


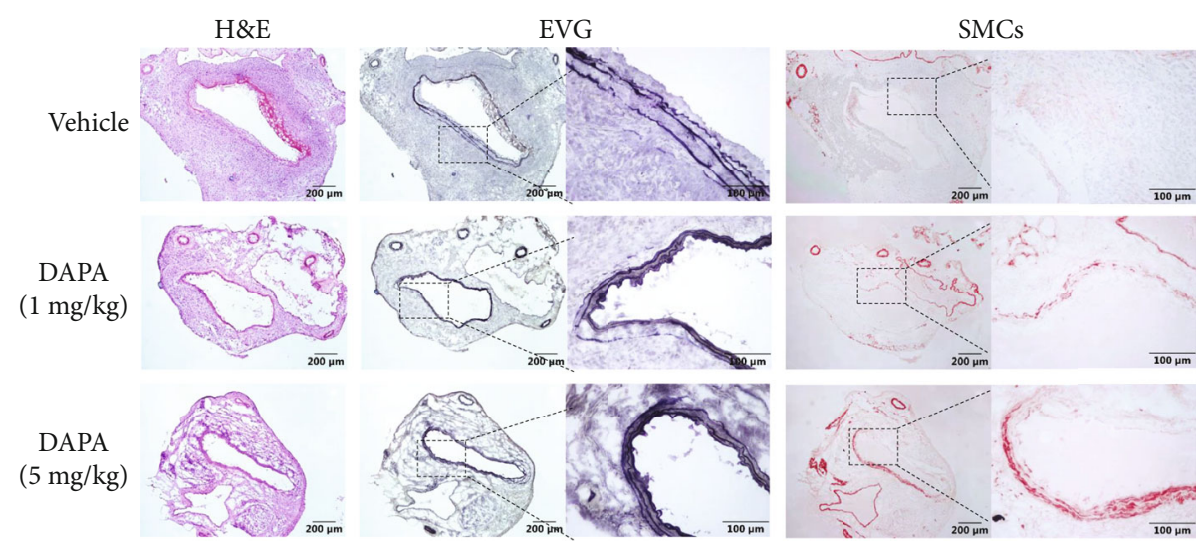

(a)

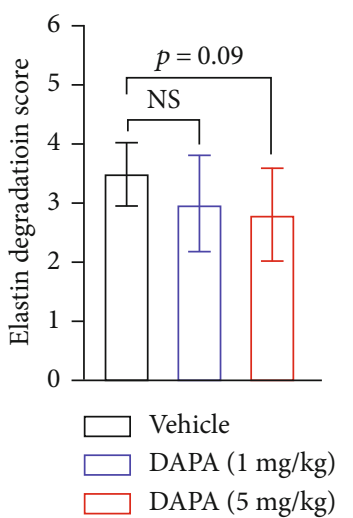

(b)

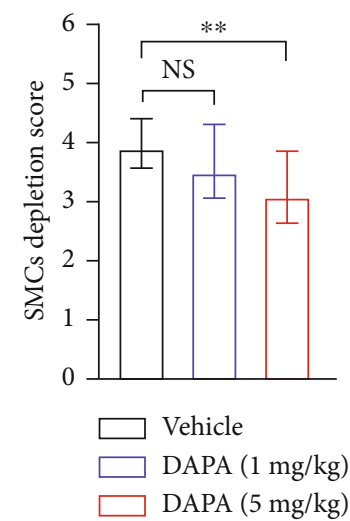

(c)

FIGURE 2: Influence of dapagliflozin treatment on medial elastin and SMC destruction. H\&E, EVG, and SMC $\alpha$-actin staining were performed on aortic frozen sections, graded, and semiquantified. (a) Representative aortic images for H\&E, medial elastin EVG staining, and SMC immunostaining. (b) and (c) Mean and SD of elastin degradation (b) and SMC depletion (c) scores. Nonparametric KruskalWallis test, ${ }^{* *} p<0.01$ between two groups, $n=10$ mice in each group. DAPA: dapagliflozin; NS: not significant.

Xi'an Jiaotong University (protocol No. 2019-1178). All animal experiments were conducted according to the Guidelines for the Care and Use of Laboratory Animals of Xi'an Jiaotong University.

2.2. Induction of Experimental AAAs. AAAs were created in male 10-12 weeks old C57BL/6 mice via intra-aortic infusion of porcine pancreatic elastase (PPE) freshly diluted in PBS for use (type I PPE, 1.5 units/mL, Table S1) as previously reported [22-26]. Briefly, mice were anesthetized by inhalation $2 \%$ isoflurane, abdominal laparotomy was created, and infrarenal aorta was exposed and infused with $30 \mu \mathrm{L}$ of PPE solution for 5 minutes under constant pressure. Thereafter, PE-10 tubing was withdrawn, aortomy was closed with 11-0 suture (Lingqiao, Ningbo, China), and aortic flow of lower limbs was restored. Following 2 hours postsurgical recovery, mice were housed in separate cages with free access to food and water.

2.3. Dapagliflozin Treatment. Dapagliflozin was purchased from Med Chem Express (Table S1) and freshly prepared by suspending in corn oil immediately prior to use. To test the effect on the formation of AAA, mice were daily treated with dapagliflozin at 1 or $5 \mathrm{mg} / \mathrm{kg}$ body weight through oral gavage $(n=10$ mice for each dose). Additional 10 mice were treated with an equal volume $(200 \mathrm{uL})$ of corn oil as vehicle. All treatments began 1 day before and terminated 14 days after PPE infusion. These doses have been proven effective in published studies [27-29]. To assess the effect on the progression of existing AAA, dapagliflozin $(5 \mathrm{mg} / \mathrm{kg})$ or an equal volume of vehicle was administered 4 days following PPE infusion, the time point by which aneurysms are formed in this model and continued for 10 days ( $n=10$ for each group) [30]. Fourteen days after PPE infusion, all mice were euthanized by carbon dioxide inhalation.

2.4. Aortic Diameter Measurement. After infrarenal aorta was exposed during surgery, abdominal aortae were immediately photographed under a microscope by a digital camera (ProS5 Lite, Motic, China) before PPE infusion. The baseline aortic diameters were measured with Images Plus 3.0 ML (Motic, China). Fourteen days following PPE infusion, aortic diameters were performed immediately prior to euthanasia. An aneurysm was defined as a more than $50 \%$ increase in aortic diameter over the baseline level [31]. 


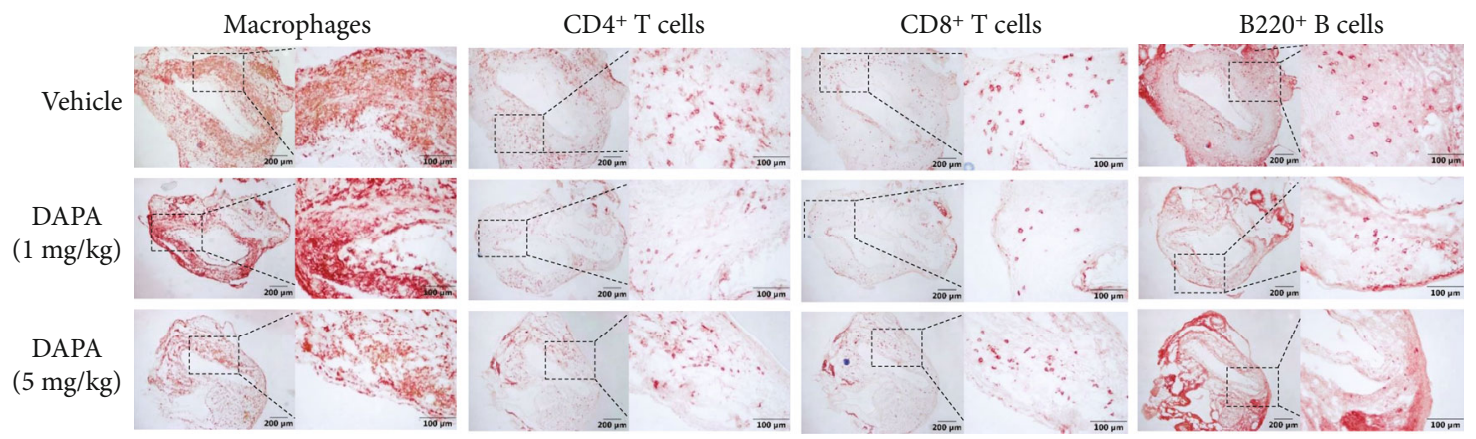

(a)

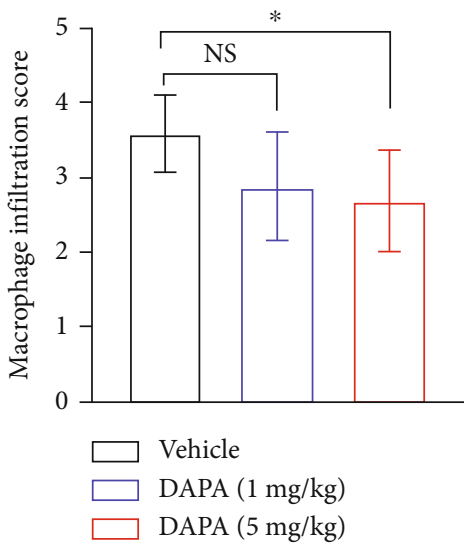

(b)

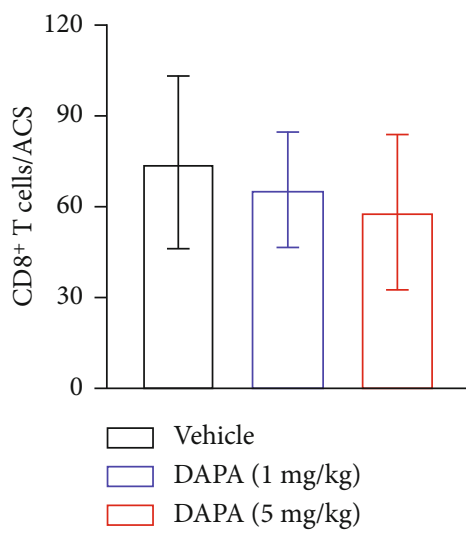

(d)

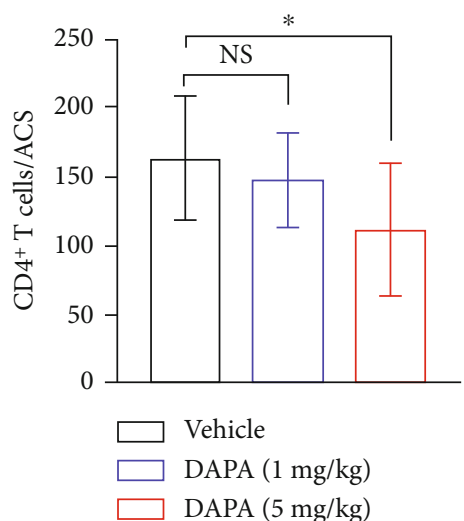

(c)

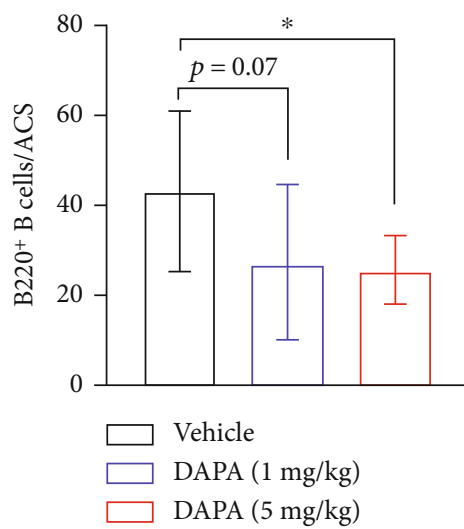

(e)

FIGURE 3: Dapagliflozin treatment attenuates aortic leukocyte infiltration. Immunostaining with antibodies against CD68, CD4, CD8, and B220 was used to evaluate the subsets of leukocytes in aneurysmal segment. (a) Immunostaining images of aortic macrophages, CD4 ${ }^{+} \mathrm{T}$ cells, and $\mathrm{CD}^{+} \mathrm{T}$ cells from PPE-infused mice treated with dapagliflozin or vehicle. (b)-(e) Quantification of different subsets of leukocytes per aortic cross-section (ACS) (mean and SD). For normally distributed data, one-way ANOVA analysis was conducted (T cells and B cells analysis), and for data failing normal distribution, the nonparametric Kruskal-Wallis test was performed (macrophage infiltration score). ${ }^{*} p<0.05$ vs. vehicle. DAPA: dapagliflozin; ACS: aortic cross-section. NS: not significant. 


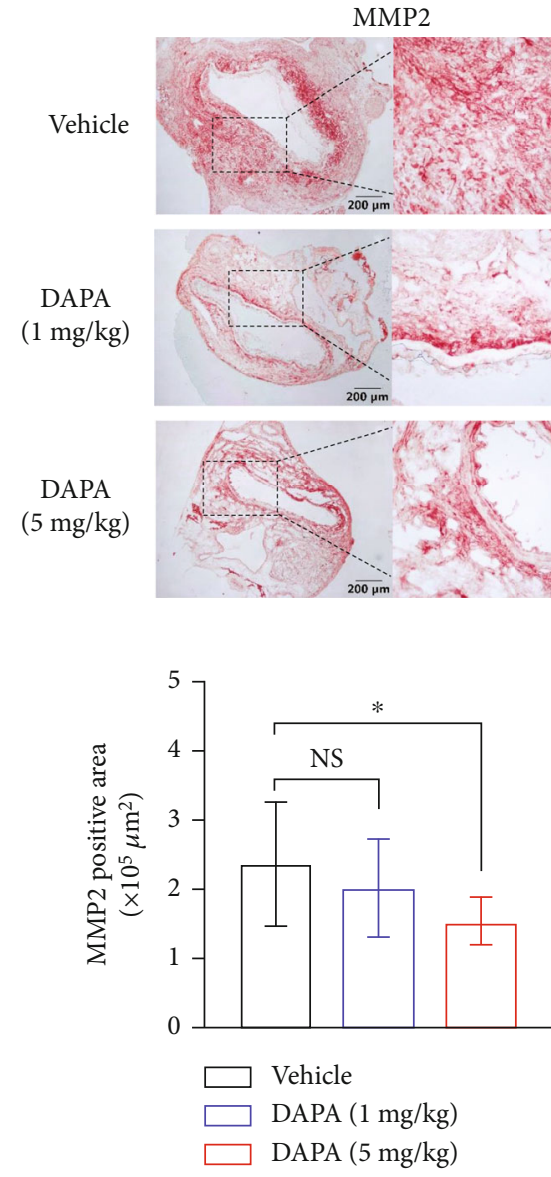

(b)

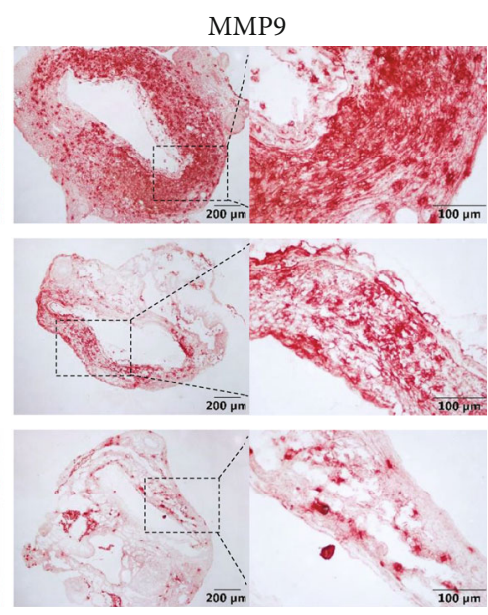

(a)

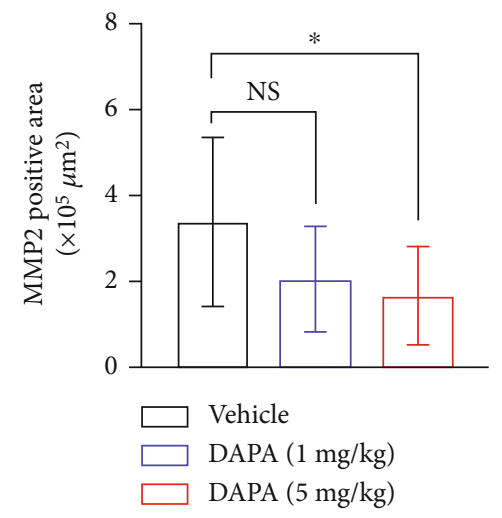

(c)

FIGURE 4: Dapagliflozin treatment reduced MMP2 and MMP9 expression levels. (a) Representative immunostaining images of MMP2 and MMP9 in aortae of PPE-infused mice treated with dapagliflozin or vehicle. (b) and (c) Quantification of MMP2 and 9 expression levels (mean and SD) in aneurysmal aorta. One-way ANOVA analysis was conducted. ${ }^{*} p<0.05$ vs. vehicle. DAPA: dapagliflozin; NS: not significant.

2.5. Histochemical Analyses. On day 14 after PPE infusion, infrarenal aortae were harvested, embedded in O.C.T. media, sectioned $(6 \mu \mathrm{m})$ and acetone-fixed. Hematoxylin and eosin (H\&E) and elastic van Gieson (EVG) staining were performed following standard or published procedure [32, 33]. Elastin degradation was graded using as previously reported [23-26, 32, 33]: grade I: elastin break or degradation was limited to one outer medial elastin layer; grade II: elastin degradation was involved in more than two layers, or entire medial elastin layer, but limited to less than $1 / 4$ of the aortic circumference (AC); grade III: elastin degradation was involved in entire medial elastin layers, but limited to less than $1 / 2$ the AC; and grade IV: elastin degradation was involved in entire elastin layers and expanded to more than $3 / 4$ of $\mathrm{AC}$ [23-25].

2.6. Immunohistochemical Analyses. Tissue immunostaining was performed on acetone-fixed frozen sections using a standard 3-step immunoperoxidase procedure as described previously $[25,33]$. Primary antibodies are antismooth muscle cells (SMCs) alpha-actin as well as rat monoclonal antibodies against $\mathrm{CD} 68$ (macrophages), $\mathrm{CD} 4\left(\mathrm{CD} 4^{+} \mathrm{T}\right.$ cells), CD8 (CD8 ${ }^{+} \mathrm{T}$ cells), B220 (B cells), and CD31 (blood vessel). T cells, B cells, and neovessels were quantified by counting positively stained cells or vessels per aortic crosssection (ACS). Medial SMC depletion and aortic macrophage accumulation were graded as I (mild) to IV (severe) as reported previously [31,32]. Additionally, the expression of aortic matrix metalloproteins (MMPs: MMP2 and MMP9) was also evaluated by immunostaining. Secondary antibodies and other key reagents included biotinylated goat antirat antibody, donkey anti-goat IgG antibody, streptavidin-peroxidase conjugate, and AEC substrate kit. All primary and secondary antibodies used are summarized in Table S1.

2.7. Statistical Analysis. All data are expressed as the mean \pm standard deviation (SD). The D'Agostino-Pearson omnibus normality test was used to determine whether individual datasets were normally distributed. For normally distributed data, one or two-way ANOVA analysis was conducted, followed by multiple comparisons for two group comparison. For data failing normal distribution, the nonparametric Kruskal-Wallis test was performed to compare three unmatched groups. For one time-point two group comparison, Student's $t$ test or nonparametric Mann-Whitney tests were used to determine statistical difference for normally 

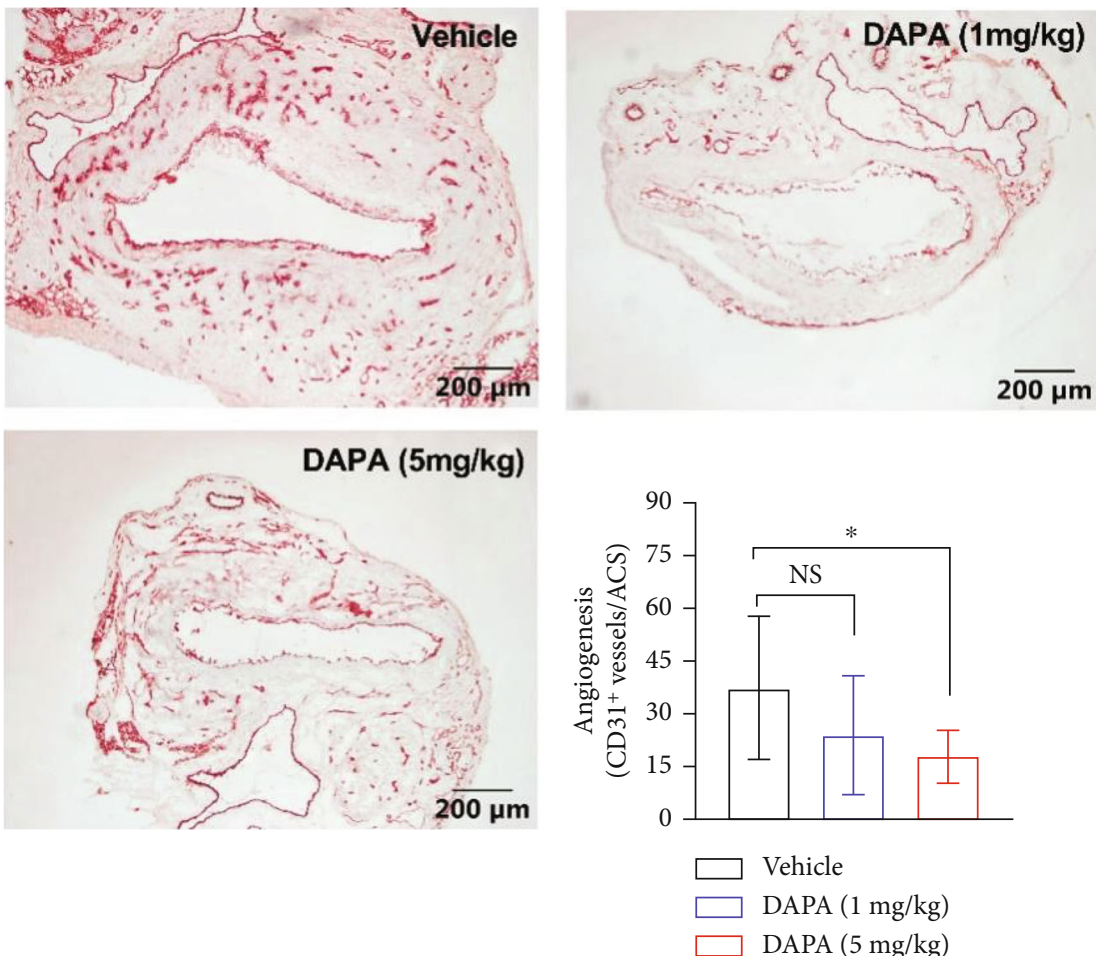

FIGURE 5: Dapagliflozin treatment attenuates aneurysmal angiogenesis. CD31 antibody staining was employed to identify neovessel in aneurysmal aorta. Quantification of mural neovessel as CD31-positive vessels per aortic cross-section. Data failing normal distribution, the nonparametric Kruskal-Wallis test was performed, ${ }^{*} p<0.05$ between two groups, $n=10$ mice in each group. ACS: aortic crosssection. DAPA: dapagliflozin; NS: not significant.

and nonnormally distributed data, respectively. All statistical analyses were performed by PRISM 7.0, and $p<0.05$ was considered significant.

\section{Results}

3.1. Dapagliflozin Treatment Ameliorates Experimental $A A A s$. To evaluate the ability of dapagliflozin to suppress experimental AAAs, mice were treated with 1 or $5 \mathrm{mg} / \mathrm{kg}$ body weight dapagliflozin or vehicle (Figure 1(a)). There was no difference in the baseline aortic diameter among three treatment groups (Figures 1(b) and 1(c)). Treatment with dapagliflozin at either dose significantly reduced aortic expansion following PPE infusion as compared to vehicle treatment especially at $5 \mathrm{mg} / \mathrm{kg}$ body weight (Figure 1). Dapagliflozin treatment at 1 and $5 \mathrm{mg} / \mathrm{kg}$ body weight led to 0.10 and $0.17 \mathrm{~mm}$ decreases in aortic diameters, respectively, as compared to vehicle treatment (Figure 1(c)). After subtracted an average aortic increase by PBS alone $(0.8 \mathrm{~mm})$ (Figure 1(b)), dapagliflozin treatment significantly reduced PPE-induced dilation (approximately 21\% and 33\% decreases in 1 or $5 \mathrm{mg} / \mathrm{kg}$ treatment groups, respectively). The change in aortic diameter was significantly smaller in mice treated with $5 \mathrm{mg} / \mathrm{kg}$ dapagliflozin than that in mice treated with vehicle (Figure 1(d)). However, the difference failed to reach statistical significance between $1 \mathrm{mg} / \mathrm{kg}$ dapagliflozin and vehicle treatment, although a reduced trend was noted $(p=0.07)$. These findings indicate that dapagliflozin treatment suppressed PPE-induced AAAs.

3.2. Dapagliflozin Treatment Preserves Medial SMCs. Medial elastin degradation and SMC loss are main histopathological features of AAAs. In Elastin and H\&E staining, although we noted a trend for reduced medial elastin destruction in dapagliflozin-, as compared to vehicle-, treated PPE-infused mice, the difference was not statistically significant (Figures 2(a) and 2(b)). In SMC alpha-actin immunohistochemical staining, dapagliflozin treatment at $5 \mathrm{mg} / \mathrm{kg}$ body weight markedly attenuated medial SMC loss as compared to vehicle treatment (Figures 2(a) and 2(c)). These results indicate that antianeurysmal effects of dapagliflozin might be in part mediated by preserving medial SMCs.

\subsection{Dapagliflozin Treatment Reduces Leukocyte Accumulation} and the Expression of MMP2 and MMP9 in Aneurysmal Aorta. Mural leukocyte accumulation is another histological hallmark and pathogenic determinant of AAAs [4-7]. Thus, we assessed the aortic infiltration of different subsets of leucocytes, including macrophages, $\mathrm{T}$ cells, and $\mathrm{B}$ cells by tissue immunostaining. In aneurysmal aorta, macrophages were the dominant infiltrating cells among all subsets evaluated (Figure 3). Compared with vehicle treatment, dapagliflozin treatment significantly reduced aortic macrophage infiltration especially at the high dose (Figures 3(a) and 3(b)). Similarly, dapagliflozin significantly reduced aortic $\mathrm{CD} 4^{+} \mathrm{T}$ cells and $\mathrm{B}$ 

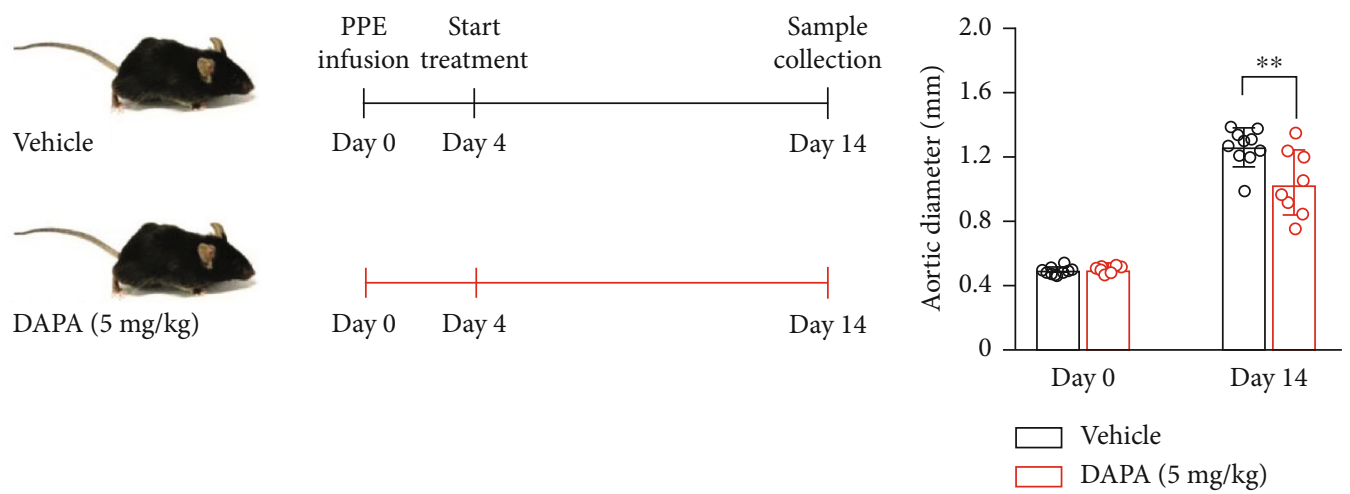

(a)

(b)

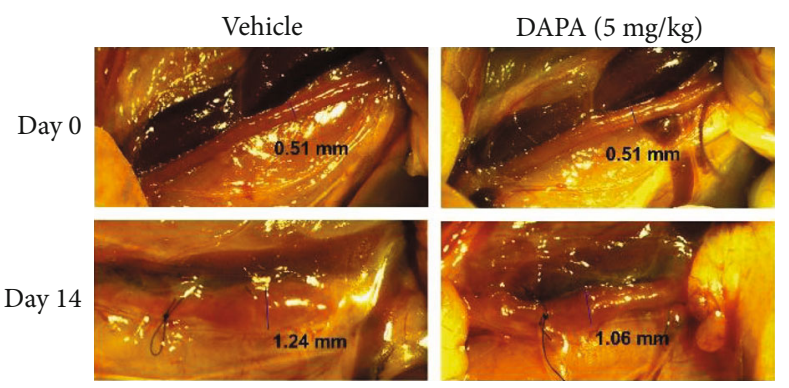

(c)

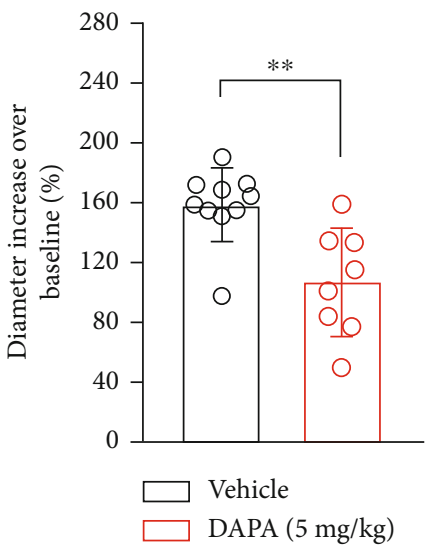

(d)

FIGURE 6: Dapagliflozin treatment limits further progression of existing AAAs. (a) treatment regimens. (b) Mean and SD of aortic diameters at the baseline level (day 0) and 14 days after PPE infusion. (c) Representative abdominal aortic images at the baseline (day 0 ) and 14 days after PPE infusion. (d) Change in aortic diameter (percent increase over the baseline level) following PPE infusions. Nonparametric MannWhitney test, ${ }^{* *} p<0.01$ compared with vehicle treatment, $n=10$ (vehicle) or 8 (dapagliflozin at $5 \mathrm{mg} / \mathrm{kg}$ ). DAPA: dapagliflozin.

cell accumulation (Figures 3(a), 3(c), and 3(e)). Although dapagliflozin treatment attenuated $\mathrm{CD} 8^{+} \mathrm{T}$ cells infiltration, there was no statistical difference between two treatment groups (Figures 3(a) and 3(d)). These results indicate that the suppression of experimental AAAs by dapagliflozin was in part mediated by inhibiting aortic macrophage accumulation. The expression of MMP2 and 9 was also diminished in aneurysmal aorta following dapagliflozin treatment as compared to vehicle treatment (Figure 4). MMPs, especially MMP2 and 9, were well known to involve in the development of AAA by regulated aortic wall remolding. The downregulation of both MMPs by dapagliflozin may be resulted from reduced MMP-producing inflammatory cell infiltration.

3.4. Dapagliflozin Treatment Suppresses Aneurysmal Angiogenesis. Because mural angiogenesis contributes to AAA pathogenesis, we determined whether the AAA suppression was associated with alteration in aortic wall angiogenesis by CD31 antibody immunostaining. Neovessel density was lower in dapagliflozin- than that in vehicletreated aneurysmal aorta (Figure 5). In high-dose dapagliflozin-treated mice, there was a $53 \%$ reduction in neovessel density as compared to vehicle-treated mice (19 vs. 37 blood vessels/ACS). These results indicate that the suppression of mural angiogenesis may also contribute to AAA suppression by dapagliflozin.

3.5. Dapagliflozin Treatment Limits Further Progression of Existing AAAs. In order to verify its clinical value, dapagliflozin at $5 \mathrm{mg} / \mathrm{kg}$ was used to treat mice with existing AAAs (Figure 6(a)). In vehicle treatment, aortic diameters continuously increased to day 14. In contrast, dapagliflozin treatment reduced the progression of existing AAAs (Figures 6(b)-6(d)). In dapagliflozin treated mice, both aortic diameters and their data changes on day 14 were significantly smaller than that in vehicle-treated mice (Figures 6(b) and 6(d)). In histological analyses, dapagliflozin treatment reduced elastin degradation, preserved medial SMCs, and attenuated aortic inflammation (Figure 7). Though the difference was not significant, dapagliflozin treatment tended to prevent the further degradation of elastin in existing AAAs (Figures 7(a) and 7(b)). Dapagliflozin treatment also significantly inhibited macrophage infiltration and improved SMC cellularity as compared to vehicle treatment (Figures 7(a), 7(c), and $7(d))$. Altogether, dapagliflozin treatment hindered further progression of aneurysmal expansion and histopathologies in mice with existing AAAs. 


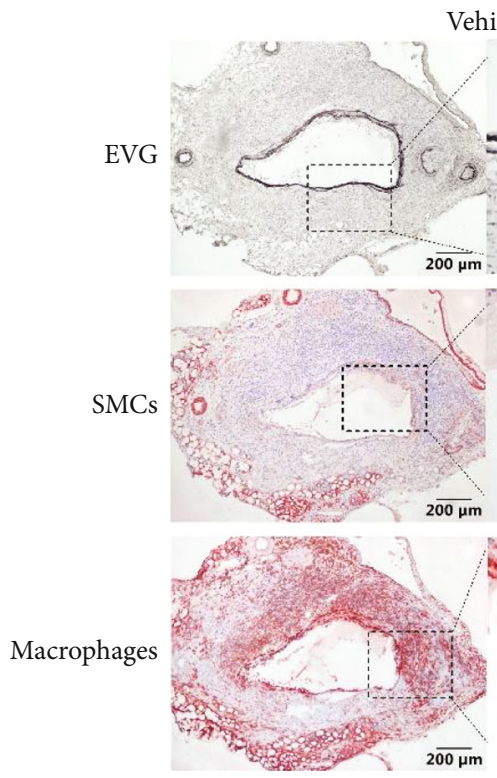

hicle
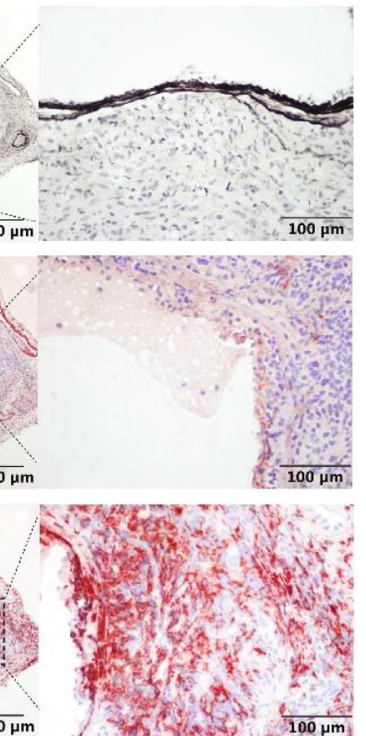

(a)

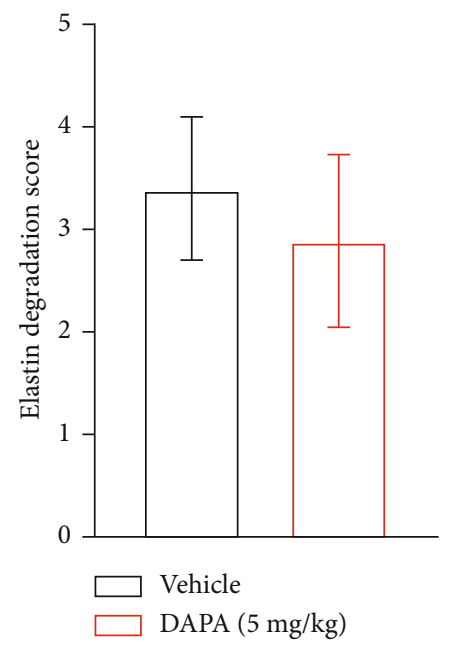

(b)

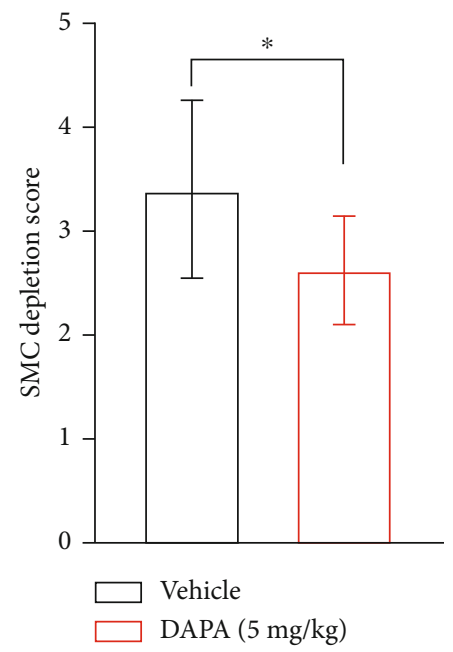

(c)
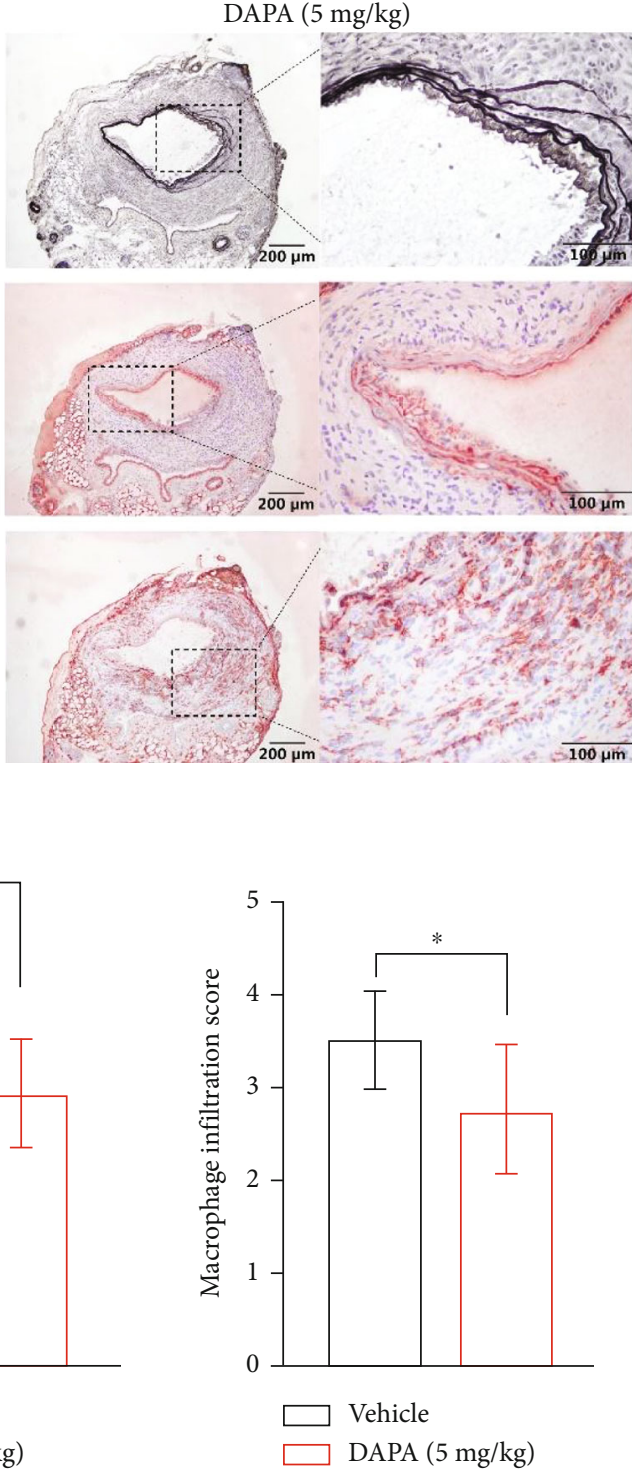

(d)

FIgURE 7: Influence of dapagliflozin on medial elastin, SMC destruction, and aortic macrophages infiltration on existing AAAs. EVG, SMC $\alpha$-actin, and CD68 (macrophage) staining were performed on aortic frozen sections, graded, and semiquantified. (a) Representative aortic images for medial elastin EVG staining, SMC immunostaining, and aortic macrophages immunostaining. (b)-(d) Mean and SD of elastin degradation score (b), SMC depletion score (c), and macrophages in aneurysmal aortae. Nonparametric Mann-Whitney test, ${ }^{*} p<0.05$ and ${ }^{* *} p<0.01$ compared with vehicle treatment, $n=10$ (vehicle) and 8 (dapagliflozin at $5 \mathrm{mg} / \mathrm{kg}$ ) mice. DAPA: dapagliflozin.

\section{Discussion}

Accumulating epidemiological studies have shown that SGLT2 inhibitors, a class of antidiabetic drugs, reduce the risk of atherosclerotic cardiovascular diseases possibly through glucose-independent mechanisms [17, 34-36]. Dapagliflozin is an FDA-approved SGLT-2 inhibitor for treating diabetes with demonstrated cardiovascular benefits in patients with diabetic renal dysfunction [37]. In patients with heart failure, dapagliflozin treatment slowed the progression of heart failure and reduced the risk of death from cardiovascular causes, regardless of diabetic status [36]. Abdominal aortic aneurysm seriously threatens life and health, and there is still a lack of effective drug therapy
[21]. The negative association of antidiabetic drugs with AAA prevalence or growth has been reported [11, 16]. Although clinical data is not available, experimental studies have found that SGLT2 inhibitors suppressed angiotensin 2 induced aortic dissecting aneurysms [19]. Current widely used experimental AAA models include induction of nondissecting AAAs or a dissecting model contained intramural rupture [38]. However, the influence of SGLT2 inhibitors on nondissecting AAAs remains to be clarified. This study found that a SGLT-2 inhibitor, dapagliflozin, limited the progression of nondissecting AAAs in normoglycemic mice.

In this study, we first explored the effect of dapagliflozin on the initiation and progression of nondissecting AAAs by administration of the drug 1 day before PPE infusion. 
Dapagliflozin at either dose significantly reduced aortic dilation as compared to vehicle treatment especially at $5 \mathrm{mg} / \mathrm{kg}$ body weight. This finding is consistent with previous study, in which empagliflozin, an alternative SGLT-2 inhibitor, suppressed angiotensin II-induced dissection AAAs in ApoE deficient mice [19]. Elastin and SMCs are the main functional components of the vascular media. PPE infusion severely degrades elastin and causes SMC apoptosis leading to media SMCs depletion $[25,39]$. In PPE infusion model, aortic leukocytes produce inflammatory cytokines, which may promote SMC apoptosis or dedifferentiation and show less alpha-actin positive staining. Dapagliflozin treatments significantly attenuated medial SMC loss and showed a trend for the preservation of elastin as compared to vehicle treatment. Another SGLT-2 inhibitor canagliflozin has been shown to inhibit intracellular glucose metabolism and promote autophagy critical for cell survival under various stress conditions [40]. We thus postulate that AAA suppression by dapagliflozin SGLT-2 inhibitor may be potentially mediated by modulating autophagy or apoptosis [41, 42]. Another explanation of protective effect of dapagliflozin treatments on SMCs and elastin may be associated with the inhibition of aortic inflammation. Dapagliflozin treatment significantly reduced aortic leucocytes infiltration, including macrophages, T cells, and B cells, following PPE infusion in mice. These results suggest that the suppression of experimental AAAs by dapagliflozin was also in part mediated by inhibiting aortic wall macrophage accumulation. SGLT-2 inhibitors have been shown to reduce the expression of proinflammatory cytokines, NLRP3, MyD88, and NF- $\kappa \mathrm{B}[19$, $43,44]$, a transcription factor regulating multiple inflammatory process. SGLT-2 inhibitors promoted antiinflammatory macrophage activation by modulating reactive oxygen and nitrogen species-dependent STAT3 pathway [45]. After intraluminal PPE infusion, the inflammation caused by it dominates the progression of AAA. Infiltrated aortic leukocytes produce elastin-degrading enzymes which may be responsible for the medial elastin degradation [46]. The suppressive effect of aortic leukocyte infiltration by dapagliflozin may also contribute to protect the elastin degradation. Macrophages contribute to the pathogenesis of AAA by producing MMPs. MMPs including MMP 1, 2, 3, 9, 12, and 13 were elevated in the aortic wall of AAA [47]. Dapagliflozin at $5 \mathrm{mg} / \mathrm{kg}$ reduced the expression levels of aortic MMP2 and MMP9, which might promote SMC survival in response to PPE infusion. AAAs are also associated with an abnormal angiogenesis as demonstrated by anti-VEGF-A therapy in experimental AAAs $[24,48]$. In this study, it was found that dapagliflozin treatment was able to reduce arterial adventitia angiogenesis. The interaction of abnormal angiogenesis and other inflammatory responses may be attenuated by dapagliflozin. Taken together, suppressing aortic inflammation including mural anagiogenesis may be major mechanisms by which dapagliflozin suppresses experimental AAAs in the PPE-induced AAA model.

To further evaluate the translational value of dapagliflozin in AAAs, the effect on existing AAA was investigated. Usually, aneurysms are formed after 4 days following PPE infusion in mice [30]. Similar with pretreatment experi- ment, posttreatment of dapagliflozin significantly limited the expansion of abdominal aortic diameter in PPEinfused mice. These findings indicate that dapagliflozin confined the progression of existing AAAs and may hold the potential for clinical AAA management. The inhibition of aortic inflammation and SMC-protective effect may play important role in this process. All the above results suggest that administration of dapagliflozin in diabetic patients, like the use of metformin, may contribute to the negative association between diabetes mellitus and AAA prevalence and growth $[11,15]$. Therefore, to investigate the protective effect of dapagliflozin on aneurysms, further clinical 1 studies are needed.

Our study has some limitations. First, this study was carried out in normoglycemic mice, and dapagliflozin treatment has no recognizable effect on random nonfasting blood glucose level (Figure S1). Because AAA was substantially suppressed in diabetic mice $[26,49]$, it is impractical to prove whether dapagliflozin causes further protection against AAAs in hyperglycemic conditions. Second, neutrophils are critical at the early stage of experimental AAAs [50]. Because we collected aortic specimens on day 14 following PPE infusion, neutrophils were not included in this study.

In conclusion, this study found that dapagliflozin treatment ameliorated elastase-induced experimental nondissecting AAAs in part by attenuating mural macrophage accumulation and neoangiogenesis. In addition to its benefits for atherosclerotic cardiovascular diseases, our research suggests that treatment with SGLT-2 inhibitors such as dapagliflozin may reduce the risk, and the progression of small AAAs.

\section{Data Availability}

Data is available on request.

\section{Conflicts of Interest}

The authors declare that there is no conflict of interest regarding the publication of this paper.

\section{Authors' Contributions}

Sihai Zhao, Baohui Xu, Enqi Liu, and Yankui Li designed this project. Haole Liu, Panpan Wei, Congcong Xia, Weilai Fu, Kangli Tian, Boyu Xu, Yafeng Li, Jiaying Sun, Yangwei $\mathrm{Xu}$, Ming $\mathrm{Lu}$, and Daxin Cheng performed animal experiments. Yali Zhang, Rong Wang, and Weirong Wang analyzed and interpreted the data. Sihai Zhao wrote the manuscript.

\section{Acknowledgments}

This work was supported by the Science Plan Project of Shaanxi Province (2020PT-004 to Sihai Zhao, 2021PT-056 to Daxin Cheng, and 2017BSHQYXMZZ18 to Yafeng Li) and the National Natural Science Foundation of China (81370379 to Sihai Zhao). 


\section{Supplementary Materials}

Table S1: reagents. Figure S1: the effect of dapagliflozin treatment on random blood glucose in nondiabetic mice. (Supplementary Materials)

\section{References}

[1] C. L. Marcaccio and M. L. Schermerhorn, "Epidemiology of abdominal aortic aneurysms," Seminars in Vascular Surgery, vol. 34, no. 1, pp. 29-37, 2021.

[2] K. C. Kent, R. M. Zwolak, N. N. Egorova et al., "Analysis of risk factors for abdominal aortic aneurysm in a cohort of more than 3 million individuals," Journal of Vascular Surgery, vol. 52, no. 3, pp. 539-548, 2010.

[3] Z. Yuan, Y. Lu, J. Wei, J. Wu, J. Yang, and Z. Cai, "Abdominal aortic aneurysm: roles of inflammatory cells," Frontiers in Immunology, vol. 11, p. 609161, 2020.

[4] M. J. Eagleton, "Inflammation in abdominal aortic aneurysms: cellular infiltrate and cytokine profiles," Vascular, vol. 20, no. 5, pp. 278-283, 2012.

[5] J. Raffort, F. Lareyre, M. Clement, R. Hassen-Khodja, G. Chinetti, and Z. Mallat, "Monocytes and macrophages in abdominal aortic aneurysm," Nature Reviews. Cardiology, vol. 14, no. 8, pp. 457-471, 2017.

[6] L. Zhang and Y. Wang, "B lymphocytes in abdominal aortic aneurysms," Atherosclerosis, vol. 242, no. 1, pp. 311-317, 2015.

[7] S. Potteaux and A. Tedgui, "Monocytes, macrophages and other inflammatory mediators of abdominal aortic aneurysm," Current Pharmaceutical Design, vol. 21, no. 28, pp. 4007-4015, 2015.

[8] D. R. Morris, P. Sherliker, R. Clack et al., "Opposite associations of aortic aneurysm with blood glucose and with diabetes mellitus," Circulation, vol. 140, no. 3, pp. 264-266, 2019.

[9] F. A. Lederle, G. R. Johnson, S. E. Wilson et al., "Prevalence and associations of abdominal aortic aneurysm detected through Screening," Annals of Internal Medicine, vol. 126, no. 6, pp. 441-449, 1997.

[10] R. T. D'cruz, I. J. Wee, N. L. Syn, and A. M. Choong, "The association between diabetes and thoracic aortic aneurysms," Journal of vascular surgery, vol. 69, no. 1, pp. 263-268.e1, 2019.

[11] N. Fujimura, J. Xiong, E. B. Kettler et al., "Metformin treatment status and abdominal aortic aneurysm disease progression," Journal of vascular surgery, vol. 64, no. 1, pp. 46-54.e8, 2016.

[12] N. K. Itoga, K. A. Rothenberg, P. Suarez et al., "Metformin prescription status and abdominal aortic aneurysm disease progression in the U.S. veteran population," Journal of vascular surgery, vol. 69, no. 3, pp. 710-716.e3, 2019.

[13] J. Raffort, R. Hassen-Khodja, E. Jean-Baptiste, and F. Lareyre, "Relationship between metformin and abdominal aortic aneurysm," Journal of Vascular Surgery, vol. 71, no. 3, pp. 10561062, 2020.

[14] J. He, N. Li, Y. Fan, X. Zhao, C. Liu, and X. Hu, "Metformin inhibits abdominal aortic aneurysm formation through the activation of the AMPK/mTOR signaling pathway," Journal of Vascular Research, vol. 58, no. 3, pp. 148-158, 2021.

[15] J. Unosson, D. Wagsater, N. Bjarnegard et al., "Metformin prescription associated with reduced abdominal aortic aneurysm growth rate and reduced chemokine expression in a Swedish cohort," Annals of Vascular Surgery, vol. 70, pp. 425-433, 2021.

[16] M. Ribeiro-Silva, J. Oliveira-Pinto, and A. Mansilha, “Abdominal aortic aneurysm: a review on the role of oral antidiabetic drugs," International Angiology, vol. 39, no. 4, pp. 330-340, 2020.

[17] T. A. Zelniker, S. D. Wiviott, I. Raz et al., "SGLT2 inhibitors for primary and secondary prevention of cardiovascular and renal outcomes in type 2 diabetes: a systematic review and metaanalysis of cardiovascular outcome trials," Lancet, vol. 393, no. 10166, pp. 31-39, 2019.

[18] Y. Fei, M. F. Tsoi, and B. M. Y. Cheung, "Cardiovascular outcomes in trials of new antidiabetic drug classes: a network meta-analysis," Cardiovascular diabetology, vol. 18, no. 1, p. 112, 2019.

[19] R. Ortega, A. Collado, F. Selles et al., "SGLT-2 (sodium-glucose cotransporter 2) inhibition reduces Ang II (angiotensin II)-induced dissecting abdominal aortic aneurysm in ApoE (apolipoprotein E) knockout mice," Arteriosclerosis, Thrombosis, and Vascular Biology, vol. 39, no. 8, pp. 1614-1628, 2019.

[20] G. Galyfos, F. Sigala, K. Mpananis et al., "Small abdominal aortic aneurysms: has anything changed so far?," Trends in Cardiovascular Medicine, vol. 30, no. 8, pp. 500-504, 2020.

[21] J. Golledge, "Abdominal aortic aneurysm: update on pathogenesis and medical treatments," Nature Reviews. Cardiology, vol. 16, no. 4, pp. 225-242, 2019.

[22] E. Sho, M. Sho, H. Nanjo, K. Kawamura, H. Masuda, and R. L. Dalman, "Hemodynamic regulation of CD34+ cell localization and differentiation in experimental aneurysms," Arteriosclerosis, Thrombosis, and Vascular Biology, vol. 24, no. 10, pp. 1916-1921, 2004.

[23] W. Wang, B. Xu, H. Xuan et al., "Hypoxia-inducible factor 1 in clinical and experimental aortic aneurysm disease," Journal of vascular surgery, vol. 68, no. 5, pp. 1538-1550, 2018.

[24] B. Xu, Y. Iida, K. J. Glover et al., "Inhibition of VEGF (vascular endothelial growth factor)-a or its receptor activity suppresses experimental aneurysm progression in the aortic elastase infusion model," Arteriosclerosis, Thrombosis, and Vascular Biology, vol. 39, no. 8, pp. 1652-1666, 2019.

[25] H. Liu, K. Tian, C. Xia et al., "Kunming mouse strain is less susceptible to elastase-induced abdominal aortic aneurysms," Animal Models and Experimental Medicine, 2021.

[26] J. Guo, T. Shoji, Y. Ge et al., "Treatment with the prolyl hydroxylase inhibitor JNJ promotes abdominal aortic aneurysm progression in diabetic mice," European Journal of Vascular and Endovascular Surgery, 2021.

[27] P. J. Millar, V. Pathak, R. C. Moffett et al., "Beneficial metabolic actions of a stable GIP agonist following pre-treatment with a SGLT2 inhibitor in high fat fed diabetic mice," Molecular and Cellular Endocrinology, vol. 420, pp. 37-45, 2016.

[28] R. Wei, X. Cui, J. Feng et al., "Dapagliflozin promotes beta cell regeneration by inducing pancreatic endocrine cell phenotype conversion in type 2 diabetic mice," Metabolism, vol. 111, p. 154324, 2020.

[29] T. Cai, Q. Ke, Y. Fang et al., "Sodium-glucose cotransporter 2 inhibition suppresses HIF- $1 \alpha$-mediated metabolic switch from lipid oxidation to glycolysis in kidney tubule cells of diabetic mice," Cell death \& disease, vol. 11, no. 5, p. 390, 2020. 
[30] H. Tanaka, B. Xu, H. Xuan et al., "Recombinant interleukin-19 suppresses the formation and progression of experimental abdominal aortic aneurysms," Journal of the American Heart Association, vol. 10, no. 17, article e022207, 2021.

[31] H. Xuan, B. Xu, W. Wang et al., "Inhibition or deletion of angiotensin II type 1 receptor suppresses elastase-induced experimental abdominal aortic aneurysms," Journal of vascular surgery, vol. 67, no. 2, pp. 573-584, 2018.

[32] T. Ikezoe, T. Shoji, J. Guo et al., "No effect of hypercholesterolemia on elastase-induced experimental abdominal aortic aneurysm progression," Biomolecules, vol. 11, no. 10, 2021.

[33] K. Tian, C. Xia, H. Liu et al., "Temporal and quantitative analysis of aortic immunopathologies in elastase-induced mouse abdominal aortic aneurysms," Journal of Immunology Research, vol. 2021, Article ID 6297332, 2021.

[34] S. D. Wiviott, I. Raz, M. P. Bonaca et al., "Dapagliflozin and cardiovascular outcomes in type 2 diabetes," The New England Journal of Medicine, vol. 380, no. 4, pp. 347-357, 2019.

[35] M. C. Petrie, S. Verma, K. F. Docherty et al., "Effect of dapagliflozin on worsening heart failure and cardiovascular death in patients with heart failure with and without diabetes," JAMA, vol. 323, no. 14, pp. 1353-1368, 2020.

[36] J. J. V. McMurray, S. D. Solomon, S. E. Inzucchi et al., "Dapagliflozin in patients with heart failure and reduced ejection fraction," New England Journal of Medicine, vol. 381, no. 21, pp. 1995-2008, 2019.

[37] T. A. Zelniker, I. Raz, O. Mosenzon et al., "Effect of Dapagliflozin on Cardiovascular Outcomes According to Baseline Kidney Function and Albuminuria Status in Patients with Type 2 Diabetes: A Prespecified Secondary Analysis of a Randomized Clinical Trial," JAMA cardiology, vol. 6, no. 7, pp. 801-810, 2021.

[38] J. Senemaud, G. Caligiuri, H. Etienne, S. Delbosc, J. B. Michel, and R. Coscas, "Translational relevance and recent advances of animal models of abdominal aortic aneurysm," Arteriosclerosis, Thrombosis, and Vascular Biology, vol. 37, no. 3, pp. 401410, 2017.

[39] P. P. Deb and A. Ramamurthi, "Spatiotemporal mapping of matrix remodelling and evidence of in situ elastogenesis in experimental abdominal aortic aneurysms," Journal of Tissue Engineering and Regenerative Medicine, vol. 11, no. 1, pp. 231-245, 2017.

[40] C. Xu, W. Wang, J. Zhong et al., "Canagliflozin exerts antiinflammatory effects by inhibiting intracellular glucose metabolism and promoting autophagy in immune cells," Biochemical Pharmacology, vol. 152, pp. 45-59, 2018.

[41] M. Packer, "Autophagy stimulation and intracellular sodium reduction as mediators of the cardioprotective effect of sodium-glucose cotransporter 2 inhibitors," European Journal of Heart Failure, vol. 22, no. 4, pp. 618-628, 2020.

[42] K. Jaikumkao, A. Pongchaidecha, N. Chueakula et al., "Dapagliflozin, a sodium-glucose co-transporter-2 inhibitor, slows the progression of renal complications through the suppression of renal inflammation, endoplasmic reticulum stress and apoptosis in prediabetic rats," Diabetes, Obesity \& Metabolism, vol. 20, no. 11, pp. 2617-2626, 2018.

[43] M. A. El-Rous, S. Saber, E. M. Raafat, and A. A. E. Ahmed, "Dapagliflozin, an SGLT2 inhibitor, ameliorates acetic acidinduced colitis in rats by targeting NF $\kappa \mathrm{B} / \mathrm{AMPK} / \mathrm{NLRP} 3$ axis," Inflammopharmacology, vol. 29, no. 4, pp. 1169-1185, 2021.
[44] V. Quagliariello, M. De Laurentiis, D. Rea et al., "The SGLT-2 inhibitor empagliflozin improves myocardial strain, reduces cardiac fibrosis and pro-inflammatory cytokines in nondiabetic mice treated with doxorubicin," Cardiovascular diabetology, vol. 20, no. 1, p. 150, 2021.

[45] T. M. Lee, N. C. Chang, and S. Z. Lin, "Dapagliflozin, a selective SGLT2 inhibitor, attenuated cardiac fibrosis by regulating the macrophage polarization via STAT3 signaling in infarcted rat hearts," Free Radical Biology \& Medicine, vol. 104, pp. 298310, 2017.

[46] M. J. Buckmaster, J. A. Curci, P. R. Murray et al., "Source of elastin-degrading enzymes in mycotic aortic aneurysms: bacteria or host inflammatory response?," Cardiovascular Surgery, vol. 7, no. 1, pp. 16-26, 1999.

[47] S. W. Rabkin, "The role matrix metalloproteinases in the production of aortic aneurysm," Progress in Molecular Biology and Translational Science, vol. 147, pp. 239-265, 2017.

[48] M. M. Thompson, L. Jones, A. Nasim, R. D. Sayers, and P. R. Bell, "Angiogenesis in abdominal aortic aneurysms," European Journal of Vascular and Endovascular Surgery, vol. 11, no. 4, pp. 464-469, 1996.

[49] N. Miyama, M. M. Dua, J. J. Yeung et al., "Hyperglycemia limits experimental aortic aneurysm progression," Journal of Vascular Surgery, vol. 52, no. 4, pp. 975-983, 2010.

[50] M. Spinosa, G. Su, M. D. Salmon et al., "Resolvin D1 decreases abdominal aortic aneurysm formation by inhibiting NETosis in a mouse model," Journal of Vascular Surgery, vol. 68, no. 6S, pp. 93S-103S, 2018. 